\title{
Caesarean scar endometriosis: a rare site of extrapelvic endometriosis
}

\author{
Sipra Bagchi $^{1 *}$, Ashish K. Gupta ${ }^{2}$, Kimmi Alwadhi ${ }^{1}$, Shanti Sah ${ }^{1}$
}

\begin{abstract}
${ }^{1}$ Department of Obstetrics \& Gynaecology, ${ }^{2}$ Department of Radiodiagnosis, SRMS Institute of Medical Sciences, Bareilly, Uttar Pradesh, India
\end{abstract}

Received: 03 August 2015

Revised: 04 August 2015

Accepted: 14 August 2015

\author{
*Correspondence: \\ Dr. Sipra Bagchi, \\ E-mail: drsiprabagchi@gmail.com
}

Copyright: ( $)$ the author(s), publisher and licensee Medip Academy. This is an open-access article distributed under the terms of the Creative Commons Attribution Non-Commercial License, which permits unrestricted non-commercial use, distribution, and reproduction in any medium, provided the original work is properly cited.

\begin{abstract}
Scar endometriosis is a rare presentation of extrapelvic endometriosis. A caesarean section scar is the most common site. The typical clinical presentation is that of a palpable firm subcutaneous nodule near surgical scars associated with cyclic pain and swelling during menses. It is often misdiagnosed with other abdominal wall and scar related pathological conditions. Diagnosis is mainly based upon a high index of suspicion. USG with color Doppler can clinch the diagnosis in patients with typical clinical features. FNAC may be inconclusive. MRI is the most sensitive but expensive modality to make the diagnosis. Wide local excision is the treatment of choice. We report a case of caesarean scar endometriosis and discuss about incidence, pathophysiology, diagnosis and treatment of this condition.
\end{abstract}

Keywords: Caesarean scar, Endometriosis, Extrapelvic

\section{INTRODUCTION}

Endometriosis was first described by Rokitansky in 1860 . It is defined as an ectopic implantation and proliferation of endometrial tissue outside the uterine cavity, commonest site being the pelvis. ${ }^{1}$ It affects approximately $10-15 \%$ of women of reproductive age. ${ }^{2}$ This incidence could be as high as $44 \%$ in asymptomatic women undergoing laparoscopy for nongynecologic symptoms. ${ }^{3}$ Extrapelvic endometriosis is less common. The possible sites are, in order of frequency, the abdominal wall, bowel, peritoneum, omentum, umbilicus, the hernia sacs, bladder, kidney, lungs, pleura and the extremities. ${ }^{2}$

Abdominal wall endometriosis often develop in previous surgical scars. Majority of the scar endometriosis are seen after obstetrical or gynaecological procedures. ${ }^{4}$ The most common site is at a caesarean section scar. ${ }^{1}$ Scar endometriosis is rare and difficult to diagnose, often confused with other surgical conditions. ${ }^{1}$ We present a case of caesarean scar endometriosis.

\section{CASE REPORT}

A 28 year old $\mathrm{P}_{2} \mathrm{~L}_{2}$, presented with a tender swelling at the left end of previous pfannensteil caesarean scar for 4 years. She had a vaginal delivery 8 years back followed by term caesarean section 4 years back for foetal distress. Patient gave history of excessive pain and increase in the size of the swelling during menstruation. Her menstrual cycles were regular. On physical examination a tender and firm subcutaneous mass of $4 \times 4 \mathrm{~cm}$ was felt at the left angle of caesarean scar. The overlying skin was puckered and adhered with underlying tissue. Rest of the scar was healthy. Ultrasonography of the mass showed an irregular hypoechoic mass in the subcutaneous plane.

On the basis of peculiar history and examination findings supported by ultrasonographic features, provisional diagnosis of scar endometriosis was made. Consequently a complete local excision of the mass was done. The mass was in the subcutaneous tissue just above the rectus sheath. Histopathology of the excised mass revealed endometrial glands and stroma interspersed within fibroadipose tissue. So a final diagnosis of scar 
endometriosis was made. Postoperative recovery was uneventful. Patient was followed for one year and there was no recurrence of the disease.

\section{DISCUSSION}

Extrapelvic endometriosis is a rare condition .It has been described to occur almost everywhere in the body, the most common site being the abdominal wall. ${ }^{3}$ The actual incidence of abdominal wall endometriosis is unknown. In one series, the prevalence of surgically proven endometriosis in scars was reported to be $1.6 \%$. $^{1}$

Scar endometriosis develop near surgical or procedural scars, such as hysterotomy, caesarean delivery, hysterectomy, appendectomy, laparoscopic trocar tract, needle tract of amniocentesis, episiotomy and tubal ligation. ${ }^{3,4}$ Incidence of scar endometriosis following hysterotomy is $1.08-2 \%$ which is more than the incidence after caesarean section i.e. $0.03-0.4 \%{ }^{4}$ The more pleuripotential capabilities of the early decidua result in cellular replication, giving rise to higher incidence of scar endometriomas after hystertomy. ${ }^{4}$ Scar endometriosis is more common in the abdominal skin and subcutaneous tissue compared to muscle and fascia. Endometriosis involving only the rectus muscle and sheath is very rare. ${ }^{1}$ The simultaneous occurrence of pelvic endometriosis has been found to be infrequent. ${ }^{1}$ The typical clinical presentation is that of a palpable firm subcutaneous nodule near surgical scars associated with cyclic pain and swelling during menses. ${ }^{3}$ It may take 3 months to 10 years after operation to develop as reported in different series. ${ }^{4}$ The most accepted theory to explain this phenomenon is that endometrial cells may be transported to distant locations, forming endometriomas during either surgical or procedural maneuvers. ${ }^{3}$ It is often misdiagnosed as keloids, haematoma, stitch granuloma, inguinal hernia, lipoma, abscess, cyst, incisional hernia, desmoid tumour, sarcoma, lymphoma, or primary and metastatic cancer in the absence of typical symptoms. 1,2

This uncommon condition can be diagnosed by various methods. In one of the case series of 12 patients by Francica et al. the sonographic morphologic findings along with vascular characteristics as shown on color Doppler examinations were described. Typical sonographic pattern was that of a subcutaneous discrete nodule, hypoechoic with scattered hyperechoic strands and irregular, often spiculated, margins infiltrating the muscularis fascia, circumscribed by a complete or incomplete hyperechoic ring caused by a perilesional inflammatory reaction. ${ }^{3}$ In the typical case, a single peripheral vascular pedicle with arterial flow entering the nodule can be shown by color Doppler. ${ }^{3}$ The authors opine that sonographic and color Doppler when combined with clinical data may substantially contribute to the preoperative diagnosis of scar endometriosis. FNAC can make cytodiagnosis of endometriosis but often it may be inconclusive as reported by Dwivedi et al. ${ }^{5} \mathrm{CT}$ or MRI can help in making diagnosis in doubtful cases when USG or FNAC is inconclusive. CT usually shows a well-defined soft tissue nodule with heterogeneous post-contrast enhancement and streakiness in the surrounding tissue. MRI is the most sensitive imaging modality but is expensive. It often accurately locates the lesion in relation to a previous caesarean section scar, with signal characteristics similar to that of background endometriosis. MRI can be more helpful when the lesion is small and is better than CT scan in detecting the planes between muscles and abdominal subcutaneous tissue. ${ }^{4}$

Hormone therapy, with oral contraceptives, progesterone and gonadotropin-releasing hormone analogues, has been found to be not effective and gives only partial relief in symptoms. Recurrence is common on cessation of therapy. ${ }^{4,6}$ Wide excision of the lesion is the mainstay of treatment for immediate relief of symptoms and prevention of recurrence. ${ }^{3,4}$ Histopathology of excised lesion should be done to confirm the diagnosis. Follow up of patients after excision is required because of the chances of recurrence. ${ }^{1}$

\section{CONCLUSION}

A high index of suspicion of scar endometriosis should be made when a woman presents with a painful swelling with or without cyclical variation in the abdominal scar especially after gynaecological or obstetrical surgery. This condition can be confused with other surgical conditions. The sonography and color Doppler along with proper correlation with clinical features is sufficient to make a preoperative diagnosis of scar endometriosis. Hormone therapy is not helpful with a higher chance of recurrence after cessation of therapy. Wide excision is the treatment of choice. Patient should be followed-up for recurrence.

\section{Funding: No funding sources Conflict of interest: None declared Ethical approval: Not required}

\section{REFERENCES}

1. Khalifa Al-Jabri Endometriosis at Caesarian Section Scar; Oman Med J. 2009;24(4):294-5.

2. Iria Neri et.al. Diagnosis and Treatment of Postcaesarean Scar Endometriosis. Acta DermatoVenereologica. Volume 87, Issue 5, Pages:428-429.

3. Giampiero Francica, MD, et.al. Abdominal Wall Endometriomas near Cesarean Delivery Scars, Sonographic and Color Doppler Findings in a Series of 12 Patients Journal of Ultrasound in Medicine. 2003;22(10):1041-7.

4. P Goel et.al. Cesarean scar endometriosis - Report of two cases. Indian journal of medical sciences. 2005;59(11):495-8.

5. Dwivedi AJ, Agrawal SN, Silva YJ. Abdominal wall endometriomas. Dig Dis Sci. 2002;47:456-61. 
6. Pikoulis E. et al. Abdominal scar endometriosis after caesarean section: report of five cases. West Indian Med J. 2011;60(3).
Cite this article as: Bagchi S, Gupta AK, Alwadhi K, Sah S. Caesarean scar endometriosis: a rare site of extrapelvic endometriosis. Int J Reprod Contracept Obstet Gynecol 2015;4:1639-41. 